\title{
The role of the CXCL12-CXCR4/CXCR7 axis in the progression and metastasis of bone sarcomas (Review)
}

\author{
YU-XIN LIAO ${ }^{1}$, CHENG-HAO ZHOU ${ }^{1}$, HUI ZENG ${ }^{1}$, DONG-QING ZUO ${ }^{1}$, ZHUO-YING WANG $^{1}$, \\ FEI YIN ${ }^{1}$, YING-QING HUA ${ }^{1}$ and ZHENG-DONG CAI ${ }^{2}$ \\ ${ }^{1}$ Shanghai Bone Cancer Institute and ${ }^{2}$ Department of Orthopaedics, Shanghai Tenth People's Hospital, \\ Tongji University School of Medicine, Shanghai 200072, P.R. China
}

Received July 8, 2013; Accepted September 25, 2013

DOI: $10.3892 /$ ijmm.2013.1521

\begin{abstract}
Bone sarcomas, which comprise less than 1\% of all human malignancies, are a group of relatively rare mesenchymal-derived tumors. They are mainly composed of osteosarcoma, chondrosarcoma and Ewing's sarcoma. In spite of advances in adjuvant chemotherapy and wide surgical resection, prognosis remains poor due to the high propensity for lung metastasis, which is the leading cause of mortality in patients with bone sarcomas. Chemokines are a superfamily of small pro-inflammatory chemoattractant cytokines which can bind to specific $\mathrm{G}$ protein-coupled seven-span transmembrane receptors. Chemokine 12 (CXCL12), also designated as stromal cell-derived factor-1 (SDF-1), is able to bind to its cognate receptors, chemokine receptor 4 (CXCR4) and chemokine receptor 7 (CXCR7), with high affinity. The binding of CXCL12 to CXCR4/CXCR7 stimulates the activation of several downstream signaling path-
\end{abstract}

Correspondence to: Professor Ying-Qing Hua, Shanghai Bone Cancer Institute, Shanghai Tenth People's Hospital, Tongji University School of Medicine, 301 Yanchang Road, Shanghai 200072,P.R. China E-mail: yingqi.hua@yahoo.com

Professor Zheng-Dong Cai, Department of Orthopaedics, Shanghai Tenth People's Hospital, Tongji University School of Medicine, 301 Yanchang Road, Shanghai 200072, P.R. China

E-mail: zhdcai@gmail.com

Abbreviations: CXCL12, chemokine 12; SDF-1, stromal cell-derived factor-1; CXCR4, chemokine receptor 4; CXCR7, chemokine receptor 7; PI3K, phosphoinositide 3-kinase; MAPK, mitogen-activated protein kinase; OS, osteosarcoma; HIF-1 $\alpha$, hypoxia-inducible factor-1 $\alpha$; CAFs, carcinoma-associated fibroblasts; G-CSF, granulocyte colony-stimulating factor; MMP-9, matrix metalloproteinase-9; VHL, von Hippel-Lindau; RCC, renal cell carcinoma; NSCLC, nonsmall cell lung cancer; VEGF, vascular endothelial growth factor; TGF- $\beta$, transforming growth factor- $\beta$; IL-5, interleukin-5; IFN- $\gamma$, interferon- $\gamma$; IL-17A, interleukin-17A; NF- $\mathrm{B}$, nuclear factor- $\kappa \mathrm{B}$; ERK, extracellular signal-regulated kinase; JNK, c-Jun N-terminal kinase; IKK $\alpha \beta$, I $\kappa$ B kinase $\alpha \beta$; hUCB-MSCs, human umbilical cord blood-mesenchymal stem cells; YY1, Yin Yang 1

Key words: chemokines, chemokine receptors, chemokine 12, chemokine receptor 4 , chemokine receptor 7 , progression, metastasis, bone sarcoma ways that regulate tumor progression and metastasis. In this review, the structure and function of CXCL12 and its receptors, CXCR4 and CXCR7, as well as many factors affecting their expression are discussed. Phosphoinositide 3-kinase (PI3K) and mitogen-activated protein kinase (MAPK) pathways are the two most important downstream pathways regulated by the CXCL12-CXCR4/CXCR7 interaction. CXCR4 expression in bone sarcomas, including tumor cells and samples and the correlation between CXCR4/CXCR7 expression and the survival of patients with bone sarcomas are also discussed. In addition, we review the involvement of the CXCL12-CXCR4/CXCR7 axis in the growth and metastasis of bone sarcomas and the targeting of this axis in preclinical studies.

\section{Contents}

1. Introduction

2. Downstream pathways involved in the CXCL12-CXCR4/ CXCR7 interaction

3. Expression of CXCR4 in bone sarcomas

4. Correlation between CXCR4/CXCR7 expression and the survival of patients with bone sarcomas

5. Involvement of the CXCL12-CXCR4/CXCR7 axis in the growth and metastasis of bone sarcomas

6. Therapies targeting the CXCL12-CXCR4/CXCR7 axis in preclinical studies

7. Conclusion

\section{Introduction}

Bone sarcomas. Sarcomas are a group of relatively rare mesenchymal-derived tumors which account for $<1 \%$ of all human malignancies $(1,2)$. They can be divided into three categories, including intermediate tumors, malignant roundcell tumors and malignant non-round-cell tumors based on differences in their biological behavior and treatment (1). Osteosarcoma (OS), chondrosarcoma and Ewing's sarcoma are the three most common tumor types in bone sarcomas.

OS is the most frequent primary malignant bone tumor, mainly occurring in children and adolescents (3). It is often localized to the distal femur and proximal tibia regions with 
a high propensity for lung metastasis, which is the leading cause of mortality and is detected in $13-27 \%$ of patients with OS at diagnosis and in $40 \%$ of patients at the developmental stage (4-6). Despite advances in adjuvant chemotherapy and surgical-wide resection, the five-year survival rate for patients with OS without and with metastases is $60-65 \%$ and $20-29 \%$, respectively (6).

Chondrosarcoma is the second most common primary malignant bone tumor which predominantly occurs in adults over 40 years of age $(7,8)$. Due to its poor response to both chemotherapy and radiotherapy, surgical resection remains an effective treatment for chondrosarcoma at present $(9,10)$. This mesenchymal malignancy has a poor prognosis with local recurrence and the five-year survival rate being $24-33 \%$ and $64-77 \%$, respectively $(11,12)$. The predilection sites of chondrosarcomas are the pelvis and femur; nevertheless, the majority of chondrosarcomas grow slowly. Although metastasis is infrequent, the lungs are still the most common metastatic site in chondrosarcomas (13-15).

Ewing's sarcoma, an aggressive round-cell sarcoma, mostly occurs in children and young adults, and is characterized by a high metastatic potential and unfavorable prognosis (16-18). The lungs and bone are the most common target organs. Approximately 25\% of patients with Ewing's sarcoma suffer from metastatic disease at diagnosis, which is usually associated with a fatal outcome $(16,17)$.

Chemokines and their receptors. Chemokines are a superfamily of 8-12-kDa chemoattractive cytokines constitutively secreted by stromal cells, including fibroblasts and endothelial cells $(19,20)$. At present, $>50$ chemokines have been identified and they can be divided into four groups $(\mathrm{C}, \mathrm{CC}, \mathrm{CXC}$ and $\mathrm{CX} 3 \mathrm{C}$ ) based on the number and position of conserved cysteines, where $\mathrm{C}$ represents the number of cysteine residues and $\mathrm{X}$ denotes the number of intervening amino acids between the conserved cysteines (21-23). Chemokines were initially discovered as essential mediators in the process of the directional migration of leukocytes to the infection and inflammation sites (24) and have been increasingly demonstrated to regulate tumor development and metastasis (25).

Chemokine receptors are $\mathrm{G}$ protein-coupled seven-transmemberane cell surface receptors to which their ligands bind with high affinity. To date, at least 20 chemokine receptors have been confirmed (22) and these receptors can also be classified into four subtypes [CXC chemokine receptors (CXCRs), $\mathrm{CC}$ chemokine receptors (CCRs), XCR and CX3CR] on the basis of their specific preference for some chemokines (26). Chemokine receptors were originally identified on leukocytes, where they have been proven to play a crucial role in inflammation (27). Not only can the same chemokines bind to different receptors, but more than one chemokine is able to bind to the same receptor to a certain extent (19). However, certain chemokines only interact with a single receptor (22). The binding of chemokines to their receptors stimulates the activation of several downstream signaling pathways that regulate tumor progression and metastasis (21).

CXCL12. Chemokine 12 (CXCL12), also designated as stromal cell-derived factor-1 (SDF-1), secreted by stromal cells including fibroblasts and endothelial cells as mentioned above is a member of the CXC subfamily of chemokines. It is widely expressed in a number of organs, such as the lungs, liver, skeletal muscle, brain, kidneys, heart, skin and bone marrow (19). Its primary role is in the homing of hematopoietic stem cells to bone marrow (28). The involvement of CXCL12 in the metastasis of various types of cancer has also been previously demonstrated (20). Increasing evidence indicates that CXCL12 can promote proliferation and survival in ovarian cancer (29), prostate cancer (30), breast cancer $(20,31,32)$, glioma (33) and glioblastoma (34). However, it has been reported that there are minimal or negligible effects on the survival and growth of myeloma in the presence of CXCL12 in vitro (35). On the one hand, the expression of CXCL12 can be affected by a number of factors. It has been reported that DNA-damaging agents, such as irradiation, cyclophosphamide, or 5-fluorouracil upregulate CXCL12 expression in mouse marrow and cultured cells (36). Hypoxia-inducible factor- $1 \alpha$ (HIF-1 $\alpha$ ) induces CXCL12 expression in hypoxic or damaged tissues (37). Besides, it is carcinoma-associated fibroblasts (CAFs) rather than normal fibroblasts that elevate CXCL12 expression $(32,38)$. Nevertheless, CXCL12 expression is reduced by granulocyte colony-stimulating factor (G-CSF) in the process of inducing hematopoietic stem cell mobilization (39). On the other hand, CXCL12 can stimulate the secretion of other factors. It has previously been demonstrated that matrix metalloproteinase-9 (MMP-9) expression is upregulated in the presence of CXCL12 when investigating the involvement of the CXCL12-CXCR4 axis in the metastasis of prostate cancer and OS $(40,41)$.

CXCR4. Chemokine receptor 4 (CXCR4), initially discovered as co-receptor facilitating the entry of T-tropic (X4) HIV viruses into $\mathrm{CD}^{+} \mathrm{T}$ cells, is the cognate receptor of CXCL12 $(42,43)$. It has been found that CXCR4 is expressed in a wide range of tissues, including brain, lymph node and small intestine tissues (21), as well as in monocytes, B cells, naïve T cells and early hematopoietic progenitor cells in the immune system (22). It should be noted that the overexpression of CXCR4 can be detected in no less than 23 different types of human cancer (44). Tumor cells expressing CXCR4 are more likely to migrate to organs with an abundant source of CXCL12 (19). Similar to CXCL12, the expression of CXCR4 is regulated by a number of factors, among which HIF- $1 \alpha$ is the most frequently mentioned. Under hypoxic conditions, the von Hippel-Lindau (VHL) tumor suppressor gene, which induces the degradation of HIF-1 is inactivated (26). Therefore, elevated levels of HIF-1 stimulate CXCR4 expression via the VHL-HIF-1 pathway in renal cell carcinoma (RCC) $(45,46)$ and non-small cell lung cancer (NSCLC) (47). The vascular endothelial growth factor (VEGF) regulated by HIF-1 can also induce CXCR4 expression in breast cancer cells (48) and glioblastoma (49). It has been confirmed that human epidermal growth factor receptor 2 (HER2)/neu detected in approximately $30 \%$ of breast cancers elevates the expression of CXCR4 by inhibiting its degradation (50). Additionally, transforming growth factor- $\beta$ (TGF- $\beta$ ) (51), interleukin-5 (IL-5) and interferon- $\gamma$ (IFN- $\gamma)$ (52) released by stromal cells and interleukin-17A (IL-17A) (53) secreted by $\mathrm{T}$ cells induce the expression of CXCR4. Of note, certain studies have reported that CXCL12 itself can alter CXCR4 expression in tumor cells. The increasing or reducing effect of CXCL12 on CXCR4 expression largely depends on the type of 


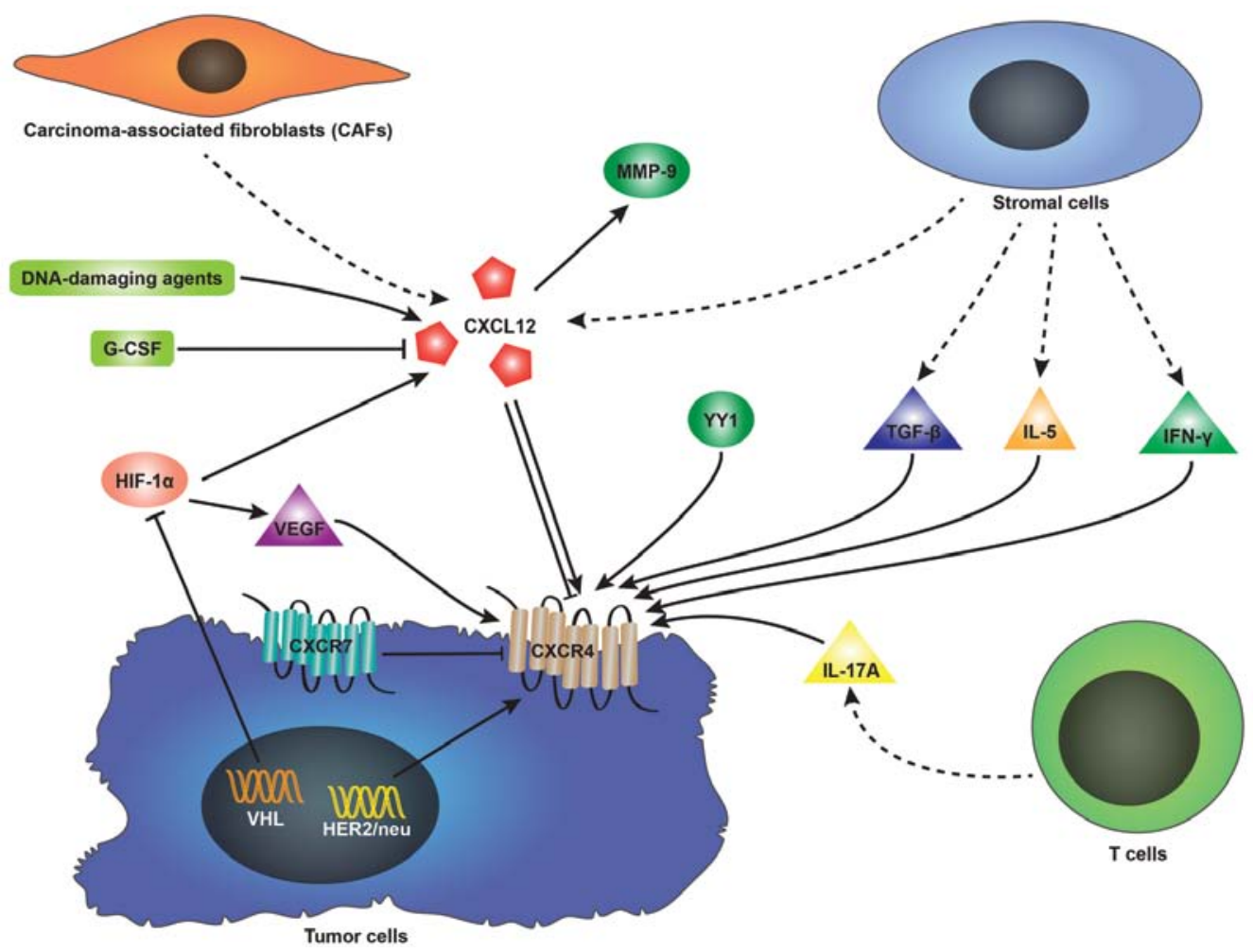

Figure 1. Factors affecting the expression of chemokine 12 (CXCL12) and chemokine receptor 4 (CXCR4). The expression of CXCL12 secreted by stromal cells and carcinoma-associated fibroblasts is upregulated by DNA-damaging agents and hypoxia-inducible factor-1 $\alpha$ (HIF-1 $\alpha$ ), whereas it is downregulated by granulocyte colony-stimulating factor (G-CSF). The expression of CXCR4 detected in tumor cells is elevated by transforming growth factor- $\beta$ (TGF- $\beta$ ), interleukin-5 (IL-5), interferon- $\gamma$ (IFN- $\gamma$ ) secreted by stromal cells and IL-17A by T cells. Von Hippel-Lindau (VHL) and HER2/neu are two genes which regulate CXCR4 expression, although with opposite effects. VHL induces the degradation of HIF-1 $\alpha$, which is the activator of the vascular endothelial growth factor (VEGF) and CXCR4. However, HER2/neu induces CXCR4 expression. Ying Yang 1 (YY1) protein is the positive regulator of CXCR4. Chemokine receptor 7 (CXCR7), the second receptor of CXCL12, inhibits CXCR4 expression. CXCL12 itself can alter CXCR4 expression and the effect is dependent on tumor type.

tumor. The surface expression of CXCR4 in oral squamous cell carcinoma (54) and OS cells (55) has been shown to be induced by CXCL12. However, Perissinotto et al (41) pointed out that CXCL12 downregulated CXCR4 expression in an OS cell line (SJSA) due to CXCR4 internalization induced by the increase in intracellular CXCR4 expression (Fig. 1).

CXCR7. CXCR4 has long been considered as the only receptor which binds to CXCL12 and regulates the biological effects induced by the CXCL12-CXCR4 pathway. However, this theory was challenged by the fact that chemokine receptor 7 (CXCR7; (RDC-1) was identified in 2005 as a novel decoy receptor of CXCL12 participating in CXCL12-CXCR4 signaling and can bind to CXCL11 (I-TAC) with low affinity $(56,57)$. Similar to CXCR4, the elevated CXCR7 expression can be detected in a number of tumors and plays an important role in promoting growth and metastasis in tumor models in vivo by regulating neoangiogenesis and organ-specific metastasis $(22,58-60)$. As opposed to the conclusions drawn by the majority of reports that CXCR7 is a positive regulator in the proliferation/metastasisenhancing effects on tumors induced by the CXCL12-CXCR4 interaction, it was revealed by Liberman et al (24) that CXCR7 eliciting anti-tumorigenic functions significantly reduced the CXCL12-CXCR4-mediated growth of CXCR7-expressing neuroblastoma cells in vitro and in vivo. Of note, in contrast to CXCR4, CXCR7 expression did not correlate with neuroblastoma grades but with tumor differentiation in their study. These results are consistent with another report that CXCR7 acts as a negative regulator of CXCR4 and abolishes the function of CXCL12 (61).

\section{Downstream pathways involved in the CXCL12-CXCR4/ CXCR7 interaction}

It is already accepted that the binding of CXCL12 to CXCR4 or CXCR7 leads to the activation of several downstream pathways that regulate cell chemotaxis, survival, proliferation and migration $(19,62,63)$. The phosphoinositide 3-kinase (PI3K) and mitogen-activated protein kinase (MAPK) pathways are most frequently investigated in related studies. The PI3K and MAPK pathways have been found to play a key role in tumor cell survival and migration $(34,40)$. PI3K activation can result in the phosphorylation of Akt, which induces the activation of nuclear factor- $\mathrm{\kappa B}(\mathrm{NF}-\kappa \mathrm{B})$ transcription factors $(19,64,65)$. MAPK pathways, including extracellular signal-regulated kinase (ERK)1/2, c-Jun N-terminal kinase (JNK) and p38 can also stimulate the expression of NF- $\mathrm{KB}$ transcription factors (66). Chinni et al (40) demonstrated that CXCL12 induced MMP-9 expression regulated by NF- $\kappa B$ activity in prostate cancer cells by activating the PI3K-Akt-NF- $\mathrm{\kappa B}$ and MEK pathways and that pre-treatment with LY294002 (PI3K inhibitor) and U0126 (MEK inhibitor) abolished the effects induced by MMP-9 through the CXCL12-CXCR4 interaction in PC-3 cells. They also suggested that PI3K may be upstream 


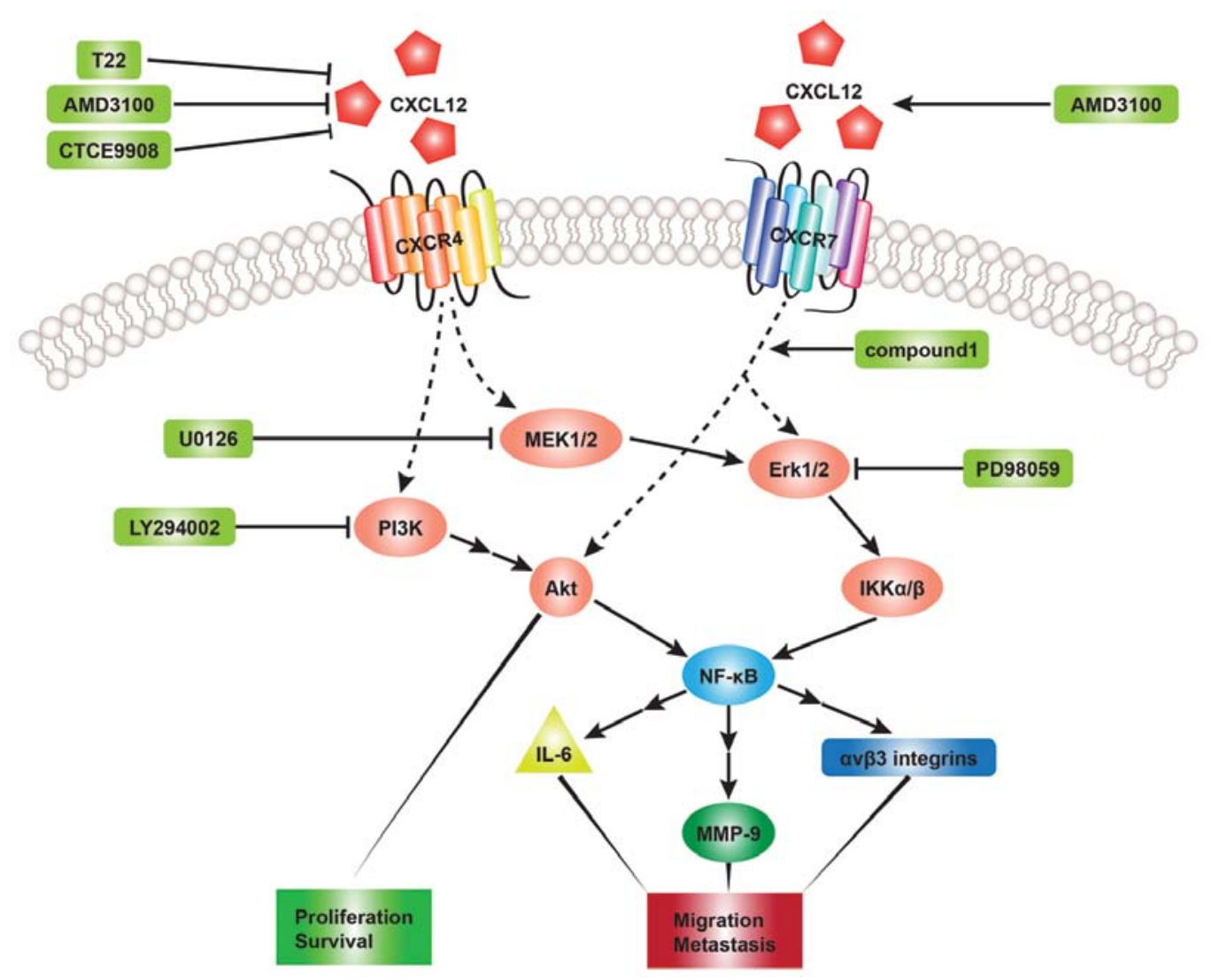

Figure 2. Downstream pathways regulated by the CXCL12-CXCR4/CXCR7 axis. The binding of chemokine 12 (CXCL12) to chemokine receptor 4 (CXCR4)

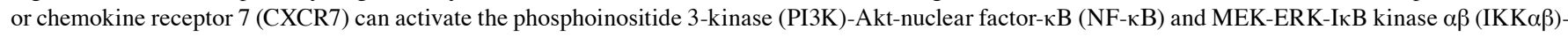
NF- $\mathrm{KB}$ pathways, which regulate both proliferation/survival and migration/metastasis in tumors. Interleukin-6 (IL-6), matrix metalloproteinase-9 (MMP-9) and $\alpha v \beta 3$ integrins induced by NF- $\mathrm{kB}$ correlate with tumor migration and metastasis. T22, AMD3100 and CTCE9908 block CXCL12-CXCR4 interaction. Nevertheless, AMD3100 is an allosteric agonist of CXCR7. CXCR7 agonist compound 1 could induces Akt and ERK phosphorylation.

of the MAPK-MEK pathway based on the result that the expression level of MMP-9 regulated by PI3K activity was significantly higher than MAPK activity. Consistent with their conclusions, Leelawat et al (67) indicated that the binding of CXCL12 to CXCR4 induced cholangiocarcinoma cell invasion by triggering the ERK1/2 and PI3K signaling pathways. The stimulation of ERK $1 / 2 / \mathrm{I} \kappa \mathrm{B}$ kinase $\alpha \beta(\mathrm{IKK} \alpha \beta)$ and $\mathrm{NF}-\kappa \mathrm{B}$ mediated by the CXCL12-CXCR4 interaction has been shown to lead to the upregulation of interleukin-6 (IL-6), promoting osteoclastogenesis in human oral cancer cells (54). Huang et al (55) pointed out that the activation of the MEK-ERKIKK $\alpha \beta-N F-\kappa B$ pathway is involved in the CXCL12-induced migration of human OS cells and the increased expression of $\alpha v \beta 3$ integrins, which has been found to play an important role in human cancer migration and metastasis $(68,69)$. Apart from the Akt and ERK transduction pathways, p38 is also involved in the process of CXCL12-mediated migration of human umbilical cord blood-mesenchymal stem cells (hUCB-MSCs) (70). This migration induced by CXCL12 was abrogated by LY294002 (PI3K inhibitor), PD98059 (MAPK/ ERK inhibitor) and SB203580 (p38 inhibitor) (70). However, much less is known about the signaling pathways regulated by the CXCL12-CXCR7 interaction. Heinrich et al (71) observed increased ERK1/2 phosphorylation in pancreatic cancer cells expressing CXCR4 and CXCR7 following exposure to CXCL12. To further elucidate the role of CXCR7 in CXCL12- induced pathways, the CXCR7 knockdown in PC cells resulted in a markedly decreased ERK phosphorylation. Therefore, they suggested that CXCR7 mediates ERK phosphorylation in the presence of CXCL12. Another study (61) revealed that CXCR7 agonist compound 1 activated both Akt and ERK phosphorylation (Fig. 2).

\section{Expression of CXCR4 in bone sarcomas}

As described previously, it has been confirmed that higher levels of CXCR4 expression can be detected in a various types of human cancer compared with normal counterparts. To our knowledge, CXCR4 expression can be evaluated by real-time PCR, western blot analysis and flow cytometry in tumor cells, and immunohistochemical staining and tissue microarray in tumor tissues. Over the past decade, a number of studies have focused on elucidating whether CXCR4 is expressed in bone sarcomas and whether its expression level correlates with metastasis and the survival of patients with bone sarcomas. It was first reported by Laverdiere et al (72) that the mRNA expression of CXCR4 was detected in $63 \%$ of OS samples, but was detected at low levels in the cell lines by fluorescent quantitative real-time PCR. Consistent with their findings, Lin et al (73) and Baumhoer et al (74) discovered that 69.6\% (39/56) and $73 \%(159 / 219)$ of OS samples expressed CXCR4 by tissue microarray and immunohistochemistry, respectively. However, 
Table I. Expression of chemokine receptor 4 (CXCR4) in osteosarcoma non-metastatic and metastatic samples.

\begin{tabular}{|c|c|c|c|c|c|c|c|}
\hline \multirow[b]{2}{*}{ Authors/(Refs.) } & \multicolumn{2}{|c|}{ CXCR4-negative } & \multicolumn{2}{|c|}{ CXCR4-positive } & \multicolumn{3}{|c|}{ CXCR4-positive (\%) } \\
\hline & No metastasis & Metastasis & No metastasis & Metastasis & No metastasis & Metastasis & Total \\
\hline Oda et al (76) & 20 & 10 & 10 & 20 & 33.30 & 66.70 & 50.00 \\
\hline Fan et al (75) & 3 & 6 & 8 & 2 & 72.70 & 25.00 & 52.60 \\
\hline Lin et al (73) & 12 & 5 & 13 & 26 & 52.00 & 83.90 & 69.60 \\
\hline Baumhoer et al (74) & 41 & 33 & 42 & 29 & 50.60 & 46.80 & 49.00 \\
\hline Namløs et al (6) & 3 & 2 & 1 & 16 & 25.00 & 88.90 & 77.30 \\
\hline Ma et al (77) & 3 & 3 & 48 & 9 & 94.12 & 75.00 & 90.48 \\
\hline
\end{tabular}

Perissinotto et al (41) detected CXCR4 expression in four human OS cell lines (SJSA, MG-63, HOS and U2OS) among which SJSA cells were found to express the highest levels by flow cytometry and western blot analysis. They indicated that CXCR4 expression levels in the cells was affected by culture conditions. Specifically, CXCR4 expression in confluent cells is lower than that in growing cells (41). Fan et al (75) first reported that five canine OS cells (POS, HMPOS, COS31, HOS and D17) expressed CXCR4 mRNA and protein. The comparison of different CXCR4 expression levels between primary and metastatic tumors was originally made by Oda et al (76), showing that approximately $66.6 \%$ (20/30) of metastatic tumors were positive for CXCR4 compared with only $33.3 \%(10 / 30)$ of primary ones. Based on these results, they suggested that CXCR4 expression may be associated with the metastatic progression of OS. This conclusion was supported by the results obtained in the study by Lin et al (73), namely that the percentage of CXCR4-positive samples in metastatic tumors was approximately $83.9 \%$ (26/31), whereas it was $52 \%$ $(13 / 25)$ in primary tumors. The positive correlation between CXCR4 expression and metastasis was further verified by Namløs et al (6). When making the comparison between primary tumors and metastatic ones, they observed a significantly increased CXCR4 gene expression in the metastatic samples. Of note, they demonstrated that primary samples that developed metastases later showed a higher CXCR4 expression than those that did not metastasize. Their study was the first to evaluate the different capabilities of primary OS samples to metastasize by detecting their CXCR4 expression. However, Fan et al (75) observed that 8/11 canine primary OS tumors expressed CXCR4 compared with only $2 / 8$ in pulmonary metastases. The reason why the expression level of CXCR4 in metastatic tumors was lower than primary tumors may be explained by one possibility that CXCR4 was involved in the initial step of mediating CXCR4-positive tumor cells to metastasize to remote organs, rather than the whole process and that tumor cells may reduce its expression once reaching their target organs (75). Ma et al (77) also demonstrated that there was no evidence to show the positive correlation between CXCR4 and metastasis by observing that CXCR4 was expressed in 48/51 non-metastatic and 9/12 metastatic OS samples (Table I). In addition to OS, CXCR4 expression has also been detected in chondrosarcoma (22/22) (78) and Ewing's sarcoma (28/44 in therapy-naïve and 7/15 in metastatic tumors) (16).

\section{Correlation between CXCR4/CXCR7 expression and the survival of patients with bone sarcomas}

A number of previous studies have demonstrated that CXCR4 and CXCR7 expression is associated with the poor survival of patients with bone sarcomas $(1,16,72,73,78,79)$. Lin et al $(73)$ made a comparison between the two-year survival rate of patients with OS expressing CXCR4 and those not expressing CXCR4. They found that the two-year survival rate of CXCR4positive patients $(32.4 \%)$ was significantly lower than that of CXCR4-negative ones (78.9\%). In Ewing's sarcoma, similar results were observed by Bennani-Baiti et al (79), namely that the five-year survival rate for patients with Ewing's sarcoma expressing low levels of CXCR4 and CXCR7 was 90\%, whereas for those with high CXCR4 and CXCR7 expression, the survival rate was 54.5 and $45.4 \%$, respectively. Bai et al (78) also demonstrated that CXCR4 expression levels correlated with the chondrosarcoma grade. Given the negative effects of CXCR4 expression on survival, it has been suggested that CXCR4 may be used as a potential prognostic factor in patients with bone sarcomas $(1,72,73)$. Clark et al (80) indicated that compared with traditional prognostic factors, such as metastases and response to chemotherapy usually used during the late stages of disease, CXCR4 as a novel molecular prognostic indicator may facilitate earlier diagnosis and treatment. However, certain studies have demonstrated that there was no significant correlation between CXCR4 and CXCR7 expression and survival (74,76). Baumhoer et al (74) suggested that the tenyear survival rate for CXCR4-positive and -negative patients with OS was 68 and 57\%, respectively; for CXCR7-postive and -negative patients it was 57 and $61 \%$, respectively.

\section{Involvement of the CXCL12-CXCR4/CXCR7 axis in the growth and metastasis of bone sarcomas}

The majority of studies have elucidated the role of the CXCL12-CXCR4 axis in the metastasis of a number of types of carcinoma, including bone sarcoma, as previously mentioned. The disruption of the CXCL12-CXCR4 interaction by CTCE-9908, a small peptide CXCR4 antagonist, has been shown to lead to a decrease in the metastatic potential of OS $\mathrm{K} 7 \mathrm{M} 2$ cells in vitro and in vivo (81). The downregulation of the expression of Yin Yang 1 (YY1) protein, which strongly correlates with the malignant degree of bone tumors induced 
by human SaOs-2 OS cells by small interfering RNA (siRNA), has been shown to reduce CXCR4-mediated migration in vitro (82). On the basis of the results that mice implanted with YY1-silenced SaOs-2 cells produced fewer vessels in vivo than those implanted with $\mathrm{SaOs}-2$ cells and injected with T22 peptide, a CXCR4 inhibitor, reduced the newly formed vessels in SaOs-2-bearing mice, whereas it was ineffective in decreasing vessel formation in YY1-silenced SaOs-2-bearing mice, de Nigris et al (82) suggested that YY1 was a positive regulator of both angiogenesis and CXCR4 signal transduction. In addition, they found that 9/10 SaOs-2-bearing mice developed metastases compared with only 4/10 YY1-silenced SaOs-2-bearing mice. The treatment of OS cells with CXCL12 induced migration by stimulating the MEK-ERK-IKK $\alpha / \beta$ NF- $\kappa \mathrm{B}$ pathway, which was inhibited by CXCR4-neutralizing antibody, CXCR4-specific inhibitor (AMD3100) and siRNA against CXCR4 (55). As regards tumor growth and progression mediated by the CXCL12-CXCR4/CXCR7 axis, only a few studies mention it. Miura et al (83) revealed that the ability to form tumors in vivo positively correlated with the levels of CXCR4 expression in human HOS OS cells. They determined the effect of CXCR4 expression on HOS tumor growth by injecting intradermally different HOS transfectant cells expressing CXCR4 at low, intermediate and high levels into one flank of mice and control HOS cells into the other flank of each mouse. The growth of tumors injected with cells expressing low levels of CXCR4was greater than the control cells eight and nine days after transplantation. The tumor volume derived from the cells expressing intermediate levels of CXCR4 was larger than the one derived from cells expressing low levels of CXCR4 ten and 11 days post-transplantation. The growth of cells expressing high levels of CXCR4 was significantly greater than any other group 12 and 13 days after injection. Apart from OS, Berghuis et al (16) indicated that the activation of the CXCL12-CXCR4 interaction induced the growth, rather than the metastasis of Ewing's sarcoma cells.

\section{Therapies targeting the CXCL12-CXCR4/CXCR7 axis in preclinical studies}

The CXCL12-CXCR4/CXCR7 axis is a potential target in interference, resulting in the inhibition of downstream signaling, which regulates tumor growth, survival and metastases. To our knowledge, chemokine receptor-specific antagonists, neutralizing antibodies and siRNA are the three most common methods widely utilized in related studies.

The CXCR4 antagonist, AMD3100, a small bicyclam molecule, was initially used to prevent X4-Tropic HIV-1 viruses entering CD4 ${ }^{+} \mathrm{T}$ cells via CXCR4 (84). De Clercq (85) suggested that the effective concentration range of AMD3100 used to inhibit HIV was 1-10 nM and it was not toxic to the host cells even when AMD3100 was used at concentration of up to $500 \mu \mathrm{M}$. Its safety and efficiency in stimulating hematopoeitic stem cell mobilization in patients with multiple myeloma and lymphoma has been demonstrated in clinical trials $(86,87)$. Due to the role of CXCR4 in tumor growth and/or metastasis, a number of studies have reported that the treatment of tumor cells with AMD3100 reduces the proliferation and migration induced by CXCL12 in vitro $(16,55)$. Berghuis et al (16) found that the proliferation-increasing effect of CXCL12 on CXCR4-positive
Ewing's sarcoma cells in the absence of serum was abrogated by AMD3100. Treatment of human OS cells with AMD3100 also inhibited the CXCL12-induced migration, as indicated by Huang et al (55). However, when exploring the effects of AMD3100 on survival and proliferation of two myeloma cell lines, Kim et al (88) for the first time observed that AMD3100 at a high concentration initially promoted the proliferation of myeloma cells under serum-deprived conditions for up to five days and subsequently inhibited its proliferation by blocking the binding of CXCL12 to CXCR4 in vitro. Of note, Kalatskaya et al (89) indicated that AMD3100 bound to CXCR7, as well as CXCR4, although with opposite effects, indicating that AMD3100 is an allosteric agonist of CXCR7.

siRNA is capable of inducing the constitutive inhibition of CXCR4 expression which facilitates a more precise assessment of the involvement of CXCR4 in tumor growth and metastasis (90). To determine the effects of CXCR4 knockdown by siRNA on tumor growth and metastasis in vivo, Lapteva et al (90) injected CXCR4-negative MDA-MB-231 breast cancer cells (in which CXCR4 expression was downregulated using siRNA) and CXCR4-positive MDA-MB-231 breast cancer cells into mammary fat pads of mice. They found that none of the mice implanted with CXCR4-negative cells developed tumors for up to 45 days, whereas all the mice inoculated with CXCR4-positive cells developed tumors within three weeks. These results are consistent with those of a previous study by Smith et al (31), indicating that the reduction of CXCR4 expression in murine 4T1 breast cancer cells by siRNA delayed tumor growth in mice. They also demonstrated that AMD3100 was less effective than siRNA in delaying tumor growth in vivo by blocking CXCR4 signaling; this was due to the variable antagonism of $\mathrm{CXCR} 4$ produced by the rapid decrease in plasma levels of the compound after dosing compared with the persistent inhibition of CXCR4 expression by siRNA (31).

\section{Conclusion}

Bone sarcomas primarily including chondrosarcomas and Ewing's sarcomas are a group of relatively rare mesenchymalderived tumors. Despite their low percentage in all human malignancies and advances in adjuvant chemotherapy and surgical-wide resection, prognosis remains poor, mainly due to the high propensity for lung metastasis, which is the leading cause of mortality in patients with bone sarcomas. It has been demonstrated that the CXCL12-CXCR4/CXCR7 pathway plays a pivotal role in several biological processes. CXCL12 and CXCR4 expression can be affected by a number of factors and the binding of CXCL12 to CXCR4/CXCR7 stimulates the activation of several downstream signaling pathways that regulate tumor progression and metastasis. The expression of CXCR4 is detected in bone sarcomas and is associated with the metastasis and survival of patients suffering from this type of malignancy. Therefore, CXCR4 may be used as a potential prognostic factor to facilitate earlier diagnosis and treatment in patients with bone sarcomas. CXCR7, the second CXCL12 receptor, may serve as a negative regulator of CXCR4 and plays an opposite role in CXCL12-CXCR4 interaction. The disruption of the CXCL12-CXCR4 pathway by AMD3100 and siRNA abrogates the CXCL12-induced proliferation and/ 
or metastasis of bone sarcoma cells. It is anticipated that the targeting of the CXCL12-CXCR4/CXCR7 pathway may be utilized as a promising therapeutic strategy in the near future.

\section{References}

1. Oda Y, Tateishi N, Matono H, et al: Chemokine receptor CXCR4 expression is correlated with VEGF expression and poor survival in soft-tissue sarcoma. Int J Cancer 124: 1852-1859, 2009.

2. Kim RH, Li BD and Chu QD: The role of chemokine receptor CXCR4 in the biologic behavior of human soft tissue sarcoma. Sarcoma 2011: 593708, 2011

3. Mirabello L, Troisi RJ and Savage SA: Osteosarcoma incidence and survival rates from 1973 to 2004: data from the Surveillance, Epidemiology, and End Results Program. Cancer 115: 1531-1543, 2009.

4. Mankin HJ, Hornicek FJ, Rosenberg AE, Harmon DC and Gebhardt MC: Survival data for 648 patients with osteosarcoma treated at one institution. Clin Orthop Relat Res 429: 286-291, 2004.

5. Bentzen SM: Prognostic factor studies in oncology: osteosarcoma as a clinical example. Int J Radiat Oncol Biol Phys 49. 513-518, 2001.

6. Namløs HM, Kresse SH, Müller CR, et al: Global gene expression profiling of human osteosarcomas reveals metastasis-associated chemokine pattern. Sarcoma 2012: 639038, 2012.

7. Clark JC, Akiyama T, Dass CR and Choong PF: New clinically relevant, orthotopic mouse models of human chondrosarcoma with spontaneous metastasis. Cancer Cell Int 10: 20, 2010.

8. Hemmati M, Abbaspour A, Alizadeh AM, et al: Rat xenograft chondrosarcoma development by human tissue fragment. Exp Oncol 33: 52-54, 2011

9. Li TM, Lin TY, Hsu SF, et al: The novel benzimidazole derivative, MPTB, induces cell apoptosis in human chondrosarcoma cells. Mol Carcinog 50: 791-803, 2011.

10. Bergh P, Gunterberg B, Meis-Kindblom JM and Kindblom LG: Prognostic factors and outcome of pelvic, sacral, and spinal chondrosarcomas: a center-based study of 69 cases. Cancer 91 : $1201-1212,2001$

11. Fiorenza F, Abudu A, Grimer RJ, et al: Risk factors for survival and local control in chondrosarcoma of bone. J Bone Joint Surg Br 84: 93-99, 2002.

12. Bruns J, Elbracht $M$ and Niggemeyer O: Chondrosarcoma of bone: an oncological and functional follow-up study. Ann Oncol 12: 859-864, 2001.

13. Qureshi A, Ahmad Z, Azam M and Idrees R: Epidemiological data for common bone sarcomas. Asian Pac J Cancer Prev 11: 393-395, 2010

14. Gelderblom H, Hogendoorn PC, Dijkstra SD, et al: The clinical approach towards chondrosarcoma. Oncologist 13: 320-329, 2008

15. Ozaki T, Hillmann A, Linder N, Blasius S and Winkelmann W: Metastasis of chondrosarcoma. J Cancer Res Clin Oncol 122: 625-628, 1996

16. Berghuis D, Schilham MW, Santos SJ, et al: The CXCR4-CXCL12 axis in Ewing sarcoma: promotion of tumor growth rather than metastatic disease. Clin Sarcoma Res 2: 24, 2012.

17. Hauer K, Calzada-Wack J, Steiger K, et al: DKK 2 mediates osteolysis, invasiveness, and metastatic spread in Ewing sarcoma. Cancer Res 73: 967-977, 2013

18. Jin Z, Zhao C, Han X and Han Y: Wnt5a promotes ewing sarcoma cell migration through upregulating CXCR4 expression. BMC Cancer 12: 480, 2012.

19. Teicher BA and Fricker SP: CXCL12 (SDF-1)/CXCR4 pathway in cancer. Clin Cancer Res 16: 2927-2931, 2010.

20. Dewan MZ, Ahmed S, Iwasaki Y, Ohba K, Toi M and Yamamoto N: Stromal cell-derived factor-1 and CXCR4 receptor interaction in tumor growth and metastasis of breast cancer. Biomed Pharmacother 60: 273-276, 2006.

21. Wang J, Loberg R and Taichman RS: The pivotal role of CXCL12 (SDF-1)/CXCR4 axis in bone metastasis. Cancer Metastasis Rev 25: 573-587, 2006.

22. Sun X, Cheng G, Hao M, et al: CXCL12/CXCR4/CXCR7 chemokine axis and cancer progression. Cancer Metastasis Rev 29: 709-722, 2010.

23. Le Y, Zhou Y, Iribarren $P$ and Wang J: Chemokines and chemokine receptors: their manifold roles in homeostasis and disease. Cell Mol Immunol 1: 95-104, 2004.
24. Liberman J, Sartelet H, Flahaut M, et al: Involvement of the CXCR7/CXCR4/CXCL12 axis in the malignant progression of human neuroblastoma. PLoS One 7: e43665, 2012.

25. Balkwill F: Cancer and the chemokine network. Nat Rev Cancer 4: 540-550, 2004.

26. Burger JA and Kipps TJ: CXCR4: a key receptor in the crosstalk between tumor cells and their microenvironment. Blood 107: 1761-1767, 2006

27. Loetscher P, Moser B and Baggiolini M: Chemokines and their receptors in lymphocyte traffic and HIV infection. Adv Immunol 74: 127-180, 2000.

28. Aiuti A, Webb IJ, Bleul C, Springer T and Gutierrez-Ramos JC: The chemokine SDF-1 is a chemoattractant for human CD34 ${ }^{+}$ hematopoietic progenitor cells and provides a new mechanism to explain the mobilization of $\mathrm{CD} 34^{+}$progenitors to peripheral blood. J Exp Med 185: 111-120, 1997.

29. Scotton CJ, Wilson JL, Scott K, et al: Multiple actions of the chemokine CXCL12 on epithelial tumor cells in human ovarian cancer. Cancer Res 62: 5930-5938, 2002.

30. Sun YX, Wang J, Shelburne CE, et al: Expression of CXCR4 and CXCL12 (SDF-1) in human prostate cancers (PCa) in vivo. J Cell Biochem 89: 462-473, 2003.

31. Smith MC, Luker KE, Garbow JR, et al: CXCR4 regulates growth of both primary and metastatic breast cancer. Cancer Res 64 : 8604-8612, 2004.

32. Orimo A, Gupta PB, Sgroi DC, et al: Stromal fibroblasts present in invasive human breast carcinomas promote tumor growth and angiogenesis through elevated SDF-1/CXCL12 secretion. Cell 121: 335-348, 2005

33. Zhou Y, Larsen PH, Hao C and Yong VW: CXCR4 is a major chemokine receptor on glioma cells and mediates their survival. J Biol Chem 277: 49481-49487, 2002.

34. Barbero S, Bonavia R, Bajetto A, et al: Stromal cell-derived factor 1alpha stimulates human glioblastoma cell growth through the activation of both extracellular signal-regulated kinases 1/2 and Akt. Cancer Res 63: 1969-1974, 2003.

35. Hideshima T, Chauhan D, Hayashi T, et al: The biological sequelae of stromal cell-derived factor-1alpha in multiple myeloma. Mol Cancer Ther 1: 539-544, 2002.

36. Ponomaryov T, Peled A, Petit I, et al: Induction of the chemokine stromal-derived factor-1 following DNA damage improves human stem cell function. J Clin Invest 106: 1331-1339, 2000

37. Ceradini DJ, Kulkarni AR, Callaghan MJ, et al: Progenitor cell trafficking is regulated by hypoxic gradients through HIF-1 induction of SDF-1. Nat Med 10: 858-864, 2004.

38. Begley L, Monteleon C, Shah RB, Macdonald JW and Macoska JA: CXCL12 overexpression and secretion by aging fibroblasts enhance human prostate epithelial proliferation in vitro. Aging Cell 4: 291-298, 2005.

39. Petit I, Szyper-Kravitz M, Nagler A, et al: G-CSF induces stem cell mobilization by decreasing bone marrow SDF-1 and up-regulating CXCR4. Nat Immunol 3: 687-694, 2002.

40. Chinni SR, Sivalogan S, Dong Z, et al: CXCL12/CXCR4 signaling activates Akt-1 and MMP-9 expression in prostate cancer cells: the role of bone microenvironment-associated CXCL12. Prostate 66: 32-48, 2006.

41. Perissinotto E, Cavalloni G, Leone F, et al: Involvement of chemokine receptor 4/stromal cell-derived factor 1 system during osteosarcoma tumor progression. Clin Cancer Res 11: 490-497, 2005.

42. Feng Y, Broder CC, Kennedy PE and Berger EA: HIV-1 entry cofactor: functional cDNA cloning of a seven-transmembrane, G protein-coupled receptor. Science 272: 872-877, 1996.

43. Wegner SA, Ehrenberg PK, Chang G, Dayhoff DE, Sleeker AL and Michael NL: Genomic organization and functional characterization of the chemokine receptor CXCR4, a major entry co-receptor for human immunodeficiency virus type 1 . J Biol Chem 273: 4754-4760, 1998.

44. Balkwill F: The significance of cancer cell expression of the chemokine receptor CXCR4. Semin Cancer Biol 14: 171-179, 2004.

45. Schioppa T, Uranchimeg B, Saccani A, et al: Regulation of the chemokine receptor CXCR4 by hypoxia. J Exp Med 198: 1391-1402, 2003

46. Zagzag D, Krishnamachary B, Yee H, et al: Stromal cell-derived factor-1alpha and CXCR4 expression in hemangioblastoma and clear cell-renal cell carcinoma: von Hippel-Lindau loss-offunction induces expression of a ligand and its receptor. Cancer Res 65: 6178-6188, 2005. 
47. Phillips RJ, Mestas J, Gharaee-Kermani M, et al: Epidermal growth factor and hypoxia-induced expression of CXC chemokine receptor 4 on non-small cell lung cancer cells is regulated by the phosphatidylinositol 3-kinase/PTEN/AKT/mammalian target of rapamycin signaling pathway and activation of hypoxia inducible factor-1alpha. J Biol Chem 280: 22473-22481, 2005.

48. Bachelder RE, Wendt MA and Mercurio AM: Vascular endothelial growth factor promotes breast carcinoma invasion in an autocrine manner by regulating the chemokine receptor CXCR4. Cancer Res 62: 7203-7206, 2002.

49. Zagzag D, Lukyanov Y, Lan L, et al: Hypoxia-inducible factor 1 and VEGF upregulate CXCR4 in glioblastoma: implications for angiogenesis and glioma cell invasion. Lab Invest 86: 1221-1232, 2006.

50. Li YM, Pan Y, Wei Y, et al: Upregulation of CXCR4 is essential for HER2-mediated tumor metastasis. Cancer Cell 6: 459-469, 2004.

51. Ao M, Franco OE, Park D, Raman D, Williams K and Hayward SW: Cross-talk between paracrine-acting cytokine and chemokine pathways promotes malignancy in benign human prostatic epithelium. Cancer Res 67: 4244-4253, 2007.

52. Zhang L, Yeger H, Das B, Irwin MS and Baruchel S: Tissue microenvironment modulates CXCR4 expression and tumor metastasis in neuroblastoma. Neoplasia 9: 36-46, 2007.

53. Wang M, Wang L, Ren T, Xu L and Wen Z: IL-17A/IL-17RA interaction promoted metastasis of osteosarcoma cells. Cancer Biol Ther 14: 155-163, 2013.

54. Tang CH, Chuang JY, Fong YC, Maa MC, Way TD and Hung CH: Bone-derived SDF-1 stimulates IL-6 release via CXCR4, ERK and NF-kappaB pathways and promotes osteoclastogenesis in human oral cancer cells. Carcinogenesis 29: 1483-1492, 2008.

55. Huang CY, Lee CY, Chen MY, et al: Stromal cell-derived factor-1/CXCR4 enhanced motility of human osteosarcoma cells involves MEK1/2, ERK and NF-kappaB-dependent pathways. J Cell Physiol 221: 204-212, 2009.

56. Balabanian K, Lagane B, Infantino S, et al: The chemokine SDF-1/CXCL12 binds to and signals through the orphan receptor RDC1 in T lymphocytes. J Biol Chem 280: 35760-35766, 2005.

57. Burns JM, Summers BC, Wang Y, et al: A novel chemokine receptor for SDF-1 and I-TAC involved in cell survival, cell adhesion, and tumor development. J Exp Med 203: 2201-2213, 2006.

58. Miao Z, Luker KE, Summers BC, et al: CXCR7 (RDC1) promotes breast and lung tumor growth in vivo and is expressed on tumor-associated vasculature. Proc Natl Acad Sci USA 104 15735-15740, 2007.

59. Wang J, Shiozawa Y, Wang Y, et al: The role of CXCR7/RDC1 as a chemokine receptor for CXCL12/SDF-1 in prostate cancer. J Biol Chem 283: 4283-4294, 2008.

60. Kollmar O, Rupertus K, Scheuer C, et al: CXCR4 and CXCR7 regulate angiogenesis and CT26.WT tumor growth independent from SDF-1. Int J Cancer 126: 1302-1315, 2010.

61. Uto-Konomi A, McKibben B, Wirtz J, et al: CXCR7 agonists inhibit the function of CXCL12 by down-regulation of CXCR4 Biochem Biophys Res Commun 431: 772-776, 2013

62. Liekens S, Schols D and Hatse S: CXCL12-CXCR4 axis in angiogenesis, metastasis and stem cell mobilization. Curr Pharm Des 16: 3903-3920, 2010

63. Duda DG, Kozin SV, Kirkpatrick ND, Xu L, Fukumura D and Jain RK: CXCL12 (SDF1alpha)-CXCR4/CXCR7 pathway inhibition: an emerging sensitizer for anticancer therapies? Clin Cancer Res 17: 2074-2080, 2011.

64. Gustin JA, Ozes ON, Akca H, et al: Cell type-specific expression of the IkappaB kinases determines the significance of phosphatidylinositol 3-kinase/Akt signaling to NF-kappa B activation. J Biol Chem 279: 1615-1620, 2004.

65. Li Y, Chinni SR and Sarkar FH: Selective growth regulatory and pro-apoptotic effects of DIM is mediated by AKT and NF-kappaB pathways in prostate cancer cells. Front Biosci 10: 236-243, 2005

66. Katiyar SK and Meeran SM: Obesity increases the risk of UV radiation-induced oxidative stress and activation of MAPK and NF-kappaB signaling. Free Radic Biol Med 42: 299-310, 2007.

67. Leelawat K, Leelawat S, Narong $S$ and Hongeng S: Roles of the MEK1/2 and AKT pathways in CXCL12/CXCR4 induced cholangiocarcinoma cell invasion. World J Gastroenterol 13: $1561-1568,2007$.

68. Burger M, Glodek A, Hartmann T, et al: Functional expression of CXCR4 (CD184) on small-cell lung cancer cells mediates migration, integrin activation, and adhesion to stromal cells. Oncogene 22: 8093-8101, 2003.
69. Lai TH, Fong YC, Fu WM, Yang RS and Tang CH: Stromal cell-derived factor-1 increase alphavbeta3 integrin expression and invasion in human chondrosarcoma cells. J Cell Physiol 218: 334-342, 2009.

70. Ryu CH, Park SA, Kim SM, et al: Migration of human umbilica cord blood mesenchymal stem cells mediated by stromal cell-derived factor-1/CXCR4 axis via Akt, ERK, and p38 signal transduction pathways. Biochem Biophys Res Commun 398 105-110, 2010.

71. Heinrich EL, Lee W, Lu J, Lowy AM and Kim J: Chemokine CXCL12 activates dual CXCR4 and CXCR7-mediated signaling pathways in pancreatic cancer cells. J Transl Med 10: 68,2012 .

72. Laverdiere C, Hoang BH, Yang R, et al: Messenger RNA expression levels of CXCR4 correlate with metastatic behavior and outcome in patients with osteosarcoma. Clin Cancer Res 11: 2561-2567, 2005

73. Lin F, Zheng SE, Shen Z, et al: Relationships between levels of CXCR4 and VEGF and blood-borne metastasis and survival in patients with osteosarcoma. Med Oncol 28: 649-653, 2011.

74. Baumhoer D, Smida J, Zillmer S, et al: Strong expression of CXCL12 is associated with a favorable outcome in osteosarcoma. Mod Pathol 25: 522-528, 2012.

75. Fan TM, Barger AM, Fredrickson RL, Fitzsimmons D and Garrett LD: Investigating CXCR4 expression in canine appendicular osteosarcoma. J Vet Intern Med 22: 602-608, 2008.

76. Oda Y, Yamamoto H, Tamiya S, et al: CXCR4 and VEGF expression in the primary site and the metastatic site of human osteosarcoma: analysis within a group of patients, all of whom developed lung metastasis. Mod Pathol 19: 738-745, 2006.

77. Ma Q, Zhou Y, Ma B, et al: The clinical value of CXCR4, HER2 and CD44 in human osteosarcoma: A pilot study. Oncol Lett 3: 797-801, 2012.

78. Bai S, Wang D, Klein MJ and Siegal GP: Characterization of CXCR4 expression in chondrosarcoma of bone. Arch Pathol Lab Med 135: 753-758, 2011

79. Bennani-Baiti IM, Cooper A, Lawlor ER, et al: Intercohort gene expression co-analysis reveals chemokine receptors as prognostic indicators in Ewing's sarcoma. Clin Cancer Res 16: 3769-3778, 2010.

80. Clark JC, Dass CR and Choong PF: A review of clinical and molecular prognostic factors in osteosarcoma. J Cancer Res Clin Oncol 134: 281-297, 2008.

81. Kim SY, Lee CH, Midura BV, et al: Inhibition of the CXCR4/ CXCL12 chemokine pathway reduces the development of murine pulmonary metastases. Clin Exp Metastasis 25: 201-211, 2008.

82. de Nigris F, Rossiello R, Schiano C, et al: Deletion of Yin Yang 1 protein in osteosarcoma cells on cell invasion and CXCR4/angiogenesis and metastasis. Cancer Res 68: 1797-1808, 2008.

83. Miura K, Uniyal S, Leabu M, et al: Chemokine receptor CXCR4- $\beta 1$ integrin axis mediates tumorigenesis of osteosarcoma HOS cells. Biochem Cell Biol 83: 36-48, 2005.

84. Hendrix CW, Collier AC, Lederman MM, et al: Safety, pharmacokinetics, and antiviral activity of AMD3100, a selective CXCR4 receptor inhibitor, in HIV-1 infection. J Acquir Immune Defic Syndr 37: 1253-1262, 2004.

85. De Clercq E: The AMD3100 story: the path to the discovery of a stem cell mobilizer (Mozobil). Biochem Pharmacol 77: 1655-1664, 2009.

86. Devine SM, Flomenberg N, Vesole DH, et al: Rapid mobilization of CD34 ${ }^{+}$cells following administration of the CXCR4 antagonist AMD3100 to patients with multiple myeloma and non-Hodgkin's lymphoma. J Clin Oncol 22: 1095-1102, 2004.

87. Cashen A, Lopez S, Gao F, et al: A phase II study of plerixafor (AMD3100) plus G-CSF for autologous hematopoietic progenitor cell mobilization in patients with Hodgkin lymphoma. Biol Blood Marrow Transplant 14: 1253-1261, 2008

88. Kim HY, Hwang JY, Kim SW, et al: The CXCR4 antagonist AMD3100 has dual effects on survival and proliferation of myeloma cells in vitro. Cancer Res Treat 42: 225-234, 2010

89. Kalatskaya I, Berchiche YA, Gravel S, Limberg BJ, Rosenbaum JS and Heveker N: AMD3100 is a CXCR7 ligand with allosteric agonist properties. Mol Pharmacol 75: 1240-1247, 2009.

90. Lapteva N, Yang AG, Sanders DE, Strube RW and Chen SY: CXCR4 knockdown by small interfering RNA abrogates breast tumor growth in vivo. Cancer Gene Ther 12: 84-89, 2005. 